\title{
Gravitational Energy in Quadratic-Curvature Gravities
}

\author{
S. Deser* and Bayram Tekin ${ }^{\dagger}$ \\ Department of Physics, Brandeis University, Waltham, Massachusetts 02454
}

(Received 30 May 2002; published 14 August 2002)

\begin{abstract}
We define energy $(E)$ and compute its values for gravitational systems involving terms quadratic in curvature. There are significant differences, both conceptually and concretely, from Einstein theory. For $D=4$, all purely quadratic models admit constant curvature vacua with arbitrary $\Lambda$, and $E$ is the "cosmological" Abbott-Deser (AD) expression; instead, $E$ always vanishes in flat, $\Lambda=0$, background. For combined Einstein-quadratic curvature systems without explicit $\Lambda$-term vacuum must be flat space, and $E$ has the usual Arnowitt-Deser-Misner form. A $\Lambda$-term forces unique de Sitter vacuum, with $E$ the sum of contributions from Einstein and quadratic parts to the AD form. We also discuss the effects on energy definition of higher curvature terms and of higher dimension.
\end{abstract}

PACS numbers: $04.50 .+\mathrm{h}$

General relativity is no different from other effective low energy models, acquiring higher momentum corrections, of quantum or string origin, to the Einstein action. They are represented locally by higher derivative additions. Coordinate invariance implies that these local higher derivative additions consist of second and higher powers of curvatures (and their derivatives). The physics of such "improved" models, as well as of those consisting of purely higher derivative terms, is not immediately reducible to that of Einstein theory, just as addition of a cosmological (zero momentum) term profoundly changes the physics of the latter. In particular, the fundamental notion of gravitational energy is sufficiently different in the presence of a $\Lambda$ term that more than two decades elapsed before its extension was obtained [1,2]. The next step in the momentum ladder likewise requires a clear understanding of its energy. Although quadratic-curvature models, with or without Einstein or $\Lambda$ terms, have long been studied, there has been a great deal of confusion about their energy. This has been due primarily to the use of a flat, rather than the more relevant (even in the absence of a fundamental $\Lambda$ term, as we shall see) constant curvature, vacuum. In this Letter, we intend to provide a universal definition of energy, and to evaluate it in appropriate asymptotic geometries, for theories quadratic (or higher) in curvatures, with or without Einstein and cosmological components.

We will initially work in $D=4$, which, interestingly, is somewhat special. Since the Gauss-Bonnet invariant $\int d^{4} x \sqrt{-g}\left(R_{\mu \nu \rho \sigma}^{2}-4 R_{\mu \nu}^{2}+R^{2}\right)$ is purely topological and does not contribute to field equations, the generic quadratic-curvature action is

$$
16 \pi \kappa I=\int d^{4} x \sqrt{-g}\left(\alpha R^{2}+\beta R_{\mu \nu}^{2}\right) .
$$

The famous conformal (Weyl) gravity corresponds to the choice $\beta=-3 \alpha$, but has no special energy features; instead, we will see that $\beta=-4 \alpha$ is unique in this respect. The "post-Einstein" constant $\kappa$ is of dimension $\left[G L^{-2}\right]=$ $[M L]^{-1}$, leaving $(\alpha, \beta)$ dimensionless.
Let us recall that there are two necessary facets of a proper energy definition: First, identification of the "Gauss law," whose existence is guaranteed by gauge invariance; second, choice of the proper vacuum, possessing sufficient Killing symmetries with respect to which global, background gauge-invariant, generators can be defined; these will always appear as surface integrals in the asymptotic vacuum. Historically, the first application was, of course, to Einstein gravity without a cosmological term [1] whose natural vacuum is flat space with its Poincaré symmetries. The next case, cosmological gravity, is a bit more involved [2], as its vacua, de Sitter (dS) or anti-de Sitter (AdS) spaces have constant (rather than zero) curvature, necessarily dictated by the cosmological constant $\Lambda$ in the action. The relevant asymptotic symmetries are, respectively, $\mathrm{SO}(4,1)$ and $\mathrm{SO}(3,2)$, which still support appropriate generators. Consider first the pure quadratic gravity of (1). Although the equivalents of the Gauss law still exist and are still the $(0 \mu)$ components of the field equations, the choice of background is neither unique nor obvious. With conventions, signature $(-,+,+,+),\left[\nabla_{\mu}, \nabla_{\nu}\right] V_{\lambda}=$ $R_{\mu \nu \lambda}{ }^{\sigma} V_{\sigma}, R_{\mu \nu} \equiv R_{\mu \lambda \nu}{ }^{\lambda}$, the field equations are

$$
\begin{aligned}
& 2 \alpha R\left(R_{\mu \nu}-\frac{1}{4} g_{\mu \nu} R\right)+(2 \alpha+\beta)\left(g_{\mu \nu} \square-\nabla_{\mu} \nabla_{\nu}\right) R \\
& \quad+\beta \square\left(R_{\mu \nu}-\frac{1}{2} g_{\mu \nu} R\right)+2 \beta\left(R_{\mu \sigma \nu \rho}-\frac{1}{4} g_{\mu \nu} R_{\sigma \rho}\right) R^{\sigma \rho} \\
& \quad=\kappa \tau_{\mu \nu},
\end{aligned}
$$

where we have introduced a (necessarily covariantly conserved) matter source $\tau_{\mu \nu}$. Now decompose the metric into the sum of a background $\bar{g}_{\mu \nu}$ [that solves the source-free version of (2)] plus a deviation $h_{\mu \nu}$ of arbitrary strength,

$$
g_{\mu \nu} \equiv \bar{g}_{\mu \nu}+h_{\mu \nu} .
$$

As originally explained in [2], which we follow here, we separate the field equations into a part linear in $h_{\mu \nu}$ plus all the nonlinear ones; the latter are moved to the right hand side, as part of the total source $T_{\mu \nu}$ that also includes $\tau_{\mu \nu}$, thereby attaining the desired form 


$$
\mathcal{O}(\bar{g})_{\mu \nu \alpha \beta} h^{\alpha \beta}=T_{\mu \nu} .
$$

The operator $\mathcal{O}(\bar{g})$ is Hermitian and depends only on the background metric (which also moves all indices and defines the covariant derivatives $\bar{\nabla}_{\mu}$ ). It inherits both background Bianchi identity and (being Hermitian) background gauge invariance from (the Bianchi identities of) the full theory, namely, $\bar{\nabla}_{\mu} \mathcal{O}(\bar{g})^{\mu \nu \alpha \beta}=\mathcal{O}(\bar{g})^{\mu \nu \alpha \beta} \bar{\nabla}_{\alpha}=0$. As a consequence of these invariances, it is guaranteed that if the background $\bar{g}_{\mu \nu}$ is a vacuum that admits Killing vectors $\bar{\xi}_{\mu}: \bar{\nabla}_{\mu} \bar{\xi}_{\nu}+\bar{\nabla}_{\nu} \bar{\xi}_{\mu}=0$, then there are associated conserved charges, and they are expressible as surface integrals,

$$
Q^{\mu}(\bar{\xi})=\int d S_{i} \mathcal{F}^{\mu i},
$$

where $\mathcal{F}^{\mu \nu}$, an antisymmetric tensor obtained from $\mathcal{O}(\bar{g})$, depends on the specific model. This is easily verified by noting that $T_{\mu \nu}$ is both background conserved and symmetric. Hence $\bar{\nabla}_{\mu}\left(\sqrt{-\bar{g}} T^{\mu \nu} \bar{\xi}_{\nu}\right) \equiv \partial_{\mu}\left(\sqrt{-\bar{g}} T^{\mu \nu} \bar{\xi}_{\nu}\right)=0$, explicitly defining an ordinarily conserved vector current. The energy is simply that charge in (5) whose Killing vector is timelike.

It is here that the first departure from the Einstein framework occurs: the theories of (1) are scale invariant and have no unique vacuum: Any constant (or zero) curvature space provides a candidate background. For our systems (2), the detailed form of (4) about a constant curvature background reads

$$
\begin{aligned}
T_{\mu \nu}= & (2 \alpha+\beta)\left(\bar{g}_{\mu \nu} \square-\bar{\nabla}_{\mu} \bar{\nabla}_{\nu}+\Lambda g_{\mu \nu}\right) R_{L} \\
& +4 \Lambda\left(2 \alpha+\frac{\beta}{3}\right) G_{\mu \nu}^{L}+\beta \square G_{\mu \nu}^{L}-\frac{2 \beta \Lambda}{3} \bar{g}_{\mu \nu} R_{L},
\end{aligned}
$$

where $\square=\bar{g}^{\mu \nu} \bar{\nabla}_{\mu} \bar{\nabla}_{\nu}, \quad G_{\mu \nu}^{L} \equiv R_{\mu \nu}^{L}-\frac{1}{2} \bar{g}_{\mu \nu} R^{L}-\Lambda h_{\mu \nu}$ with $\bar{\nabla}^{\mu} G_{\mu \nu}^{L}=0$; we define $\bar{R}_{\mu \sigma \nu \rho}=\frac{\Lambda}{3}\left(\bar{g}_{\mu \nu} \bar{g}_{\sigma \rho}-\right.$ $\left.\bar{g}_{\mu \rho} \bar{g}_{\sigma \nu}\right)$, so that $\bar{R}_{\mu \nu}=\Lambda \bar{g}_{\mu \nu}$. The degenerate, $\Lambda=0$, case $\bar{g}_{\mu \nu}=\eta_{\mu \nu}$, just leads to $T_{\mu \nu} \rightarrow\left(\partial \partial R^{L}\right)_{\mu \nu}$, which necessarily implies that the energy of all asymptotically flat solutions of any purely quadratic model vanishes: this is an obvious aspect of the fact that equations of the form $\nabla^{4} \phi=\rho$ are solved by $\phi \rightarrow r\left[\int d^{3} x \rho\right]$ : energy and source are not related by a Poisson operator. [This remark directly accounts for the well-known result [3] that energy in Weyl gravity vanishes for asymptotically flat metrics, but it is no different in this respect from any other $(\alpha, \beta)$ system.] We emphasize that while energy is too degenerate to be meaningful here, asymptotically flat solutions are not excluded thereby, nor do Hamiltonian methods cease to be applicable, for example, in the analysis of the excitation spectrum about flat space.

We come next to the generic case of $\bar{g}_{\mu \nu}$ with $\Lambda \neq 0$. Here the linearization produces a universal effect: We find that

$$
\begin{gathered}
8 \pi \kappa Q^{\mu}(\bar{\xi})=2 \Lambda(4 \alpha+\beta) \int d^{3} x \sqrt{-\bar{g}} \bar{\xi}_{\nu} G_{L}^{\mu \nu} \\
+(2 \alpha+\beta) \int d S_{i} \sqrt{-\bar{g}}\left\{\bar{\xi}^{\mu} \bar{\nabla}^{i} R_{L}\right. \\
+R_{L} \bar{\nabla}^{\mu} \bar{\xi}^{i} \\
\left.-\bar{\xi}^{i} \bar{\nabla}^{\mu} R_{L}\right\} \\
+\beta \int d S_{i} \sqrt{-\bar{g}}\left\{\bar{\xi}_{\nu} \bar{\nabla}^{i} G_{L}^{\mu \nu}-\bar{\xi}_{\nu} \bar{\nabla}^{\mu} G_{L}^{i \nu}\right. \\
\left.-G_{L}^{\mu \nu} \bar{\nabla}^{i} \bar{\xi}_{\nu}+G_{L}^{i \nu} \bar{\nabla}^{\mu} \bar{\xi}_{\nu}\right\} .
\end{gathered}
$$

The integral in the first line is the standard charge of cosmological Einstein gravity [2], itself also a surface integral, of course. (In obtaining the above, gauge-invariant, surface form of the charge, it is helpful to organize the integrand to exhibit antisymmetry in $\mu$ and i.) Simple as this result is, it becomes even nicer when we turn to the evaluation of the relevant, exterior, asymptotic solutions, namely, the Schwarzschild-de Sitter (SdS) and Schwarzschild-anti-de Sitter metrics; we label them collectively by $\mathrm{SdS}$ for brevity. (Here a major difference between dS and AdS, that the former has an intrinsic horizon, enters. As explained in [2], the dS energy definition is strictly valid only inside the horizon, where the relevant Killing vector stays timelike: This restriction also logically entails that the black hole horizon be small compared to the cosmological one. We do not discuss the question of global definability or usefulness of dS energy [4], as it is really separate from the choice of dynamical model. No such problem affects the AdS case, where the surface integrals may be taken at spatial infinity.) In cosmological Einstein gravity, $Q^{0}=\int \bar{\xi}_{\nu} G_{L}^{0 \nu}$ indeed gives the desired value $8 \pi M G$, where $M$ is the "Schwarzschild" mass in the SdS solutions. Here we find that the extra, second and third, lines of (7) all vanish for SdS spaces, so generically the energy is proportional to that of cosmological Einstein gravity:

$$
E=4 \Lambda r_{0} \kappa^{-1}(4 \alpha+\beta),
$$

where $r_{0}$ is the coefficient of $1 / r$ in the usual static form of $\mathrm{SdS}$, i.e., the monopole moment of the total source, $\rho$, including (as always) gravitational contributions; it becomes proportional to the source mass, $m=\int d^{3} x \tau_{00}$, for weak fields and sources, just as in Einstein theory. [More precisely, the Gauss equation is of the form $\Lambda \nabla^{2} \phi=\kappa \rho$, so that $r_{0}=(\kappa / \Lambda) M$; the effective gravitational constant is $(\kappa / \Lambda)$ here.] Note that Weyl theory now has nonvanishing energy. Instead, it is the special $\beta=$ $-4 \alpha$ theory, whose action is the square of the traceless Ricci tensor $\tilde{R}_{\mu \nu}=R_{\mu \nu}-\frac{1}{4} g_{\mu \nu} R$ that has vanishing energy for all values of $\Lambda$.

We consider now the combined Einstein plus quadraticcurvature theories. If there is no explicit $\Lambda$ term, then constant curvature spaces are no longer solutions of the combined equations, and we are forced to flat background: 
consequently, the Einstein term's energy expression is the whole story (which does not mean that the quadratic terms do not contribute, as sources, to its value). If instead, a $\Lambda$ term is also present, then constant curvature with precisely that $\Lambda$ value is not only an allowed but the unique vacuum; the scale is now fixed by the Einstein part, and the energy is

$$
E=r_{0} G^{-1}+4 \Lambda r_{0} \kappa^{-1}(4 \alpha+\beta) .
$$

Here $r_{0}$ is the "Schwarzschild mass" that solves the Poisson equation, with contributions from both $R$ and $R^{2}$ parts. For weak fields and sources, $E$ reassuringly becomes proportional to $m$, as before.

Thus far, we have worked in $D=4$ and considered only models with at most quadratic terms in curvatures. Depending on its physical origins, a given higher power term may be viewed either as a part of the fundamental action (e.g., if there is no Einstein term, as in Weyl gravity) or as a small correction that should not be in the "kinetic" term, nor a defining component of the energy expressions, though it still affects their values. However, even when viewed as sources, higher curvature powers pose a problem as they can prevent the existence of nonzero curvature background vacua. Put another way, their contributions to $T_{\mu \nu}$ do not fall off at infinity. Take, for example, a generic higher curvature invariant $\int R^{n}, n>2$, or $R$ representing Riemann, Ricci, or scalar curvatures, possibly involving also (an even number of) covariant derivatives. Schematically, this gives rise to a field equation contribution of the form $\left(R^{n}\right)_{\mu \nu}+\left(\nabla \nabla R^{n-1}\right)_{\mu \nu}$. Just as for $n=2$, its linearization about flat space does not affect the energy. However, generically these terms do not allow constant curvature solutions, since they are not homogeneous of order zero in the metric: $\bar{R}_{\mu \nu}^{n}$ does not vanish even though $\bar{\nabla} \bar{\nabla}\left(\bar{R}^{n-1}\right)_{\mu \nu}=0$ does. While the linearization, $\Lambda^{n-2}[\Lambda+\bar{\nabla} \bar{\nabla}] R_{L}$, does resemble that of $n=2$, the background part $\left(\bar{R}^{n}\right)_{\mu \nu}$ acts (as mentioned above) as a constant source in the Gauss equation. If we classify the nonlinear terms according to the powers of the three basis tensorsWeyl (traceless part of Riemann), $\tilde{R}$ (traceless part of Ricci), and scalar curvature-then they will allow cosmological vacua if they are not pure $R^{n}$. They also explicitly contribute to the energy, as well as being sources of it, through the linear $h_{\mu \nu}$ terms in their field equations' expansions.

The above complications arise immediately in $D>4$ : There, the quadratic terms themselves are no longer homogeneous of degree zero in the metric, seemingly forbidding (A)dS backgrounds. There are three independent invariants (unlike the two at $D=4$ ). We may take this basis to be (no longer conformally invariant) Weyl gravity, the $\tilde{R}^{2}$ term, and (scalar) $R^{2}$. The latter action forbids constant curvature, while the first two clearly permit it (see also [5]). Hence they also support our energy definition. The $\tilde{R}^{2}$ term's explicit contribution will vanish just as it did in $D=$ 4. This leaves the Weyl gravity as both allowing nonflat vacuum and contributing explicitly to energy. The relevant asymptotic metric here is again SdS (or slightly generalized form allowed for pure Weyl theory). If Einstein and cosmological terms are also present, our earlier $D=4$ analysis applies straightforwardly.

In summary, we have defined energy for arbitrary general covariant gravitational models, particularly the simplest, quadratic-curvature, systems. In $D=4$, pure quadratic actions have useful, nonvanishing energy (only) with respect to cosmological backgrounds. While these vacua are infinitely degenerate, their value for any chosen $\Lambda$ is quite physical, being proportional to that of the Schwarzschild mass in the relevant SdS metric. Models with both Einstein and quadratic actions differ in imposing a unique background; here the total energy is the sum of contributions proportional to the cosmological (AbbottDeser) mass, if there is also an explicit $\Lambda$ term, and just equal to the Arnowitt-Deser-Misner mass if $\Lambda=0$. We also studied the effect on energy definition of higher curvature invariants and its extension to $D>4$. The general framework could be maintained if the higher powers contained curvature combinations such as Weyl tensors that vanish in (A)dS, and not just the scalar curvature. For $D>$ 4, there are three independent quadratic terms: If $R^{2}$ is present, flat vacuum is forced. The other two combinations, in particular, Weyl gravity, allow (A)dS backgrounds and hence permit $D=4$ energy construction. Details will be presented elsewhere.

This work was supported by National Science Foundation Grant No. PHY99-73935.

*Email address: deser@brandeis.edu

${ }^{\dagger}$ Email address: tekin@brandeis.edu

[1] R. Arnowitt, S. Deser, and C. Misner, Phys. Rev. 116, 1322 (1959); 117, 1595 (1960); in Gravitation: An Introduction to Current Research, edited by L. Witten (Wiley, New York, 1962).

[2] L. F. Abbott and S. Deser, Nucl. Phys. B195, 76 (1982).

[3] D. G. Boulware, G. T. Horowitz, and A. Strominger, Phys. Rev. Lett. 50, 1726 (1983).

[4] E. Witten, hep-th/0106109; A. Strominger, J. High Energy Phys. 0110, 034 (2001).

[5] M. Cvetic, S. Nojiri, and S.D. Odintsov, Nucl. Phys. B628, 295 (2002). 